\title{
Prevalence, management and outcomes of cardiac tamponade complicating 66,812 invasive cardiac procedures: single-center clinical registry
}

\author{
Maria Adamczyk ${ }^{1}$, Jacek T. Niedziela ${ }^{1}$, Jarosław Wasilewski ${ }^{1}$, Michał O. Zembala ${ }^{2}$, Zbigniew Kalarus ${ }^{3}$, \\ Mariusz Gąsior ${ }^{1}$ \\ ${ }^{1} 3^{\text {rd }}$ Department of Cardiology, Faculty of Medical Sciences in Zabrze, Medical University of Silesia in Katowice, Silesian Centre for Heart \\ Diseases, Zabrze, Poland \\ 2Department of Cardiothoracic Surgery and Transplantology, Silesian Centre for Heart Diseases, Zabrze, Poland \\ ${ }^{3}$ Department of Cardiology, Congenital Heart Diseases and Electrotherapy, Medical University of Silesia in Katowice, Silesian Centre for \\ Heart Diseases, Zabrze, Poland
}

Adv Interv Cardiol 2021; 17, 2 (64): 193-199

DOI: https://doi.org/10.5114/aic.2021.107499

\begin{abstract}
A bstract
Introduction: There are numerous studies concerning iatrogenic cardiac tamponade. Those studies are predominantly focused on one cardiac procedure and the follow-up is not always presented.

Aim: To estimate the rate of cardiac tamponade following 66,812 percutaneous invasive cardiac interventions depending on the procedure. For each group the baseline characteristics and hospital management, as well as in-hospital, 30-day and 1-year mortality, were evaluated.

Material and methods: The study was a single-center retrospective analysis performed in a tertiary clinical hospital, which encompasses two cardiology departments, assessing a large sample of patients who underwent percutaneous invasive cardiac procedures complicated with cardiac tamponade between January 2006 and December 2018. For this purpose, medical records and hospital databases were analyzed. Long-term follow-up was obtained in cooperation with the Silesian Cardiovascular Base.

Results: The rate of iatrogenic cardiac tamponade during the 13 -year period was $0.176 \%$. The incidence among selected invasive cardiac procedures ranged between $0.09 \%$ and $1.42 \%$. The majority of cases $(104 / 118)$ were treated by pericardiocentesis, 16 had pericardiotomy and 4 patients had both therapies. Inotropes were used in $25-45 \%$, blood transfusion in $45 \%$ of patients. The highest in-hospital mortality was observed in patients with cardiac tamponade after transcatheter aortic valve implantation. The highest 30-day and 1-year mortality rates were seen in the group with temporary electrode pacing.

Conclusions: The low incidence of cardiac tamponade with the high number of patients requiring intensive care supply and high in-hospital mortality tend to confirm that cardiac tamponade is a rare but life-threatening complication.
\end{abstract}

Key words: complications, cardiac tamponade, invasive cardiac procedures.

S u m m a ry

The application of invasive cardiovascular procedures with the need for transseptal puncture and intraprocedural anticoagulation has recently increased. It results in a higher risk of iatrogenic cardiac tamponade (CT), which is a life-threatening condition requiring prompt diagnosis and treatment. Many previous studies concerning CT were predominantly focused on one cardiac procedure, and the follow-up was not always presented. We analyzed the incidence, baseline characteristics, management, as well as in-hospital and long-term mortality in a large cohort of patients with iatrogenic CT during 13 years. The incidence of iatrogenic CT complications in our clinical center (Encompassing two cardiology departments) was relatively low or similar to other studies.

\section{Corresponding author:}

Maria Adamczyk MD, $3^{\text {rd }}$ Department of Cardiology, Faculty of Medical Sciences, Medical University of Silesia, Silesian Centre for Heart Diseases, 9 Marie Curie-Skłodowskiej St, 41-800 Zabrze, Poland, e-mail: maria.ester.adamczyk@gmail.com

Received: 22.01.2021, accepted: 6.03.2021. 


\section{Introduction}

In recent years, the application of invasive cardiovascular procedures and the use of antiplatelet and anticoagulation drugs have increased in clinical medicine [1] The risk of iatrogenic cardiac tamponade (CT) or pericardial effusion increases with the need for transseptal puncture and intraprocedural anticoagulation [2]. The clinical presentation of $C T$ is determined by the rate of pericardial fluid accumulation [3]. Iatrogenic $\mathrm{CT}$ is more likely to develop rapidly as a consequence of heart perforation [4]. Such an urgent situation necessitates prompt diagnosis, emergency treatment by pericardiocentesis or pericardiotomy, and often requires hemodynamic or ventilatory support, cardiopulmonary resuscitation or blood transfusion [5-7].

\section{Aim}

The aim of this study was to investigate the incidence, baseline characteristics, management, as well as in-hospital and long-term outcomes in a large cohort of patients with iatrogenic $\mathrm{CT}$ requiring drainage in a single university medical center during a 13-year period. The procedures we focused on were: atrial fibrillation ablation (AFA), percutaneous coronary intervention ( $\mathrm{PCI}$ ), a cardiovascular implantable electronic device (CIED), temporary pacing electrode (TPE), left atrial appendage closure (LAAC), transcatheter aortic valve implantation (TAVI) and endomyocardial biopsy (EMB).

\section{Material and methods}

In our single-center study from the Silesian Centre for Heart Diseases (SCCS) in Zabrze, Poland, which encompasses two cardiology departments, we analyzed all patients older than 18 who underwent a percutaneous invasive cardiac procedure and developed iatrogenic CT. For this study's purpose, significant CT was considered to

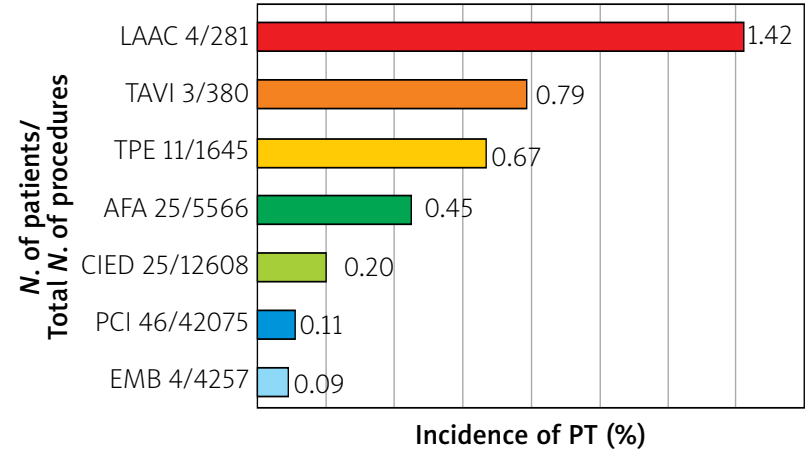

Figure 1. Incidence of pericardial tamponade after selected invasive cardiac procedures, from January 2006 to December 2018

AFA - atrial fibrillation ablation, CIED - cardiovascular implantable electronic device, EMB - endomyocardial biopsy, LAAC - left atrial appendage closure, $P C I$ - percutaneous coronary intervention, PT - pericardial tamponade, TAVI - transcatheter aortic valve implantation, TPE - temporary pacing electrode. be one that required pericardiocentesis or pericardiotomy. As the first step, we searched the internal data sets for hospital discharge with a principal diagnosis of pericardiocentesis or pericardiotomy between January 2006 and December 2018. Additionally, manual reviews were performed to exclude patients who underwent pericardial/thoracic drainage for other reasons than $\mathrm{CT}$. In the registry of 417 matching-criteria patients with different CT etiology, the iatrogenic drainage-demanded CT was considered the target population. Three patients required only conservative treatment and were not included in our analyses. Enrolled patients (118) were divided into seven groups depending on the type of cardiac intervention: AFA, PCI, CIED, TPE, LAAC, TAVI, EMB.

The medical procedures were evaluated using the ICD-9 codes. The total number of performed invasive cardiac procedures was established to calculate the incidence of CT in each group. A retrospective analysis of medical records and hospital databases, baseline characteristics, length of hospital stay, and in-hospital management for each group was performed. Data on long-term follow-up for all patients were obtained from the Silesian Cardiovascular Base formed as a result of an agreement between the $3^{\text {rd }}$ Department and the Clinical Department of Cardiology of the Medical University of Silesia, the SCCS, and the Regional-Silesian Branch of the National Health Fund in Katowice [8].

\section{Statistical analysis}

The distribution of continuous variables was tested using the Shapiro-Wilk test. None of the variables had a normal distribution. Continuous variables were presented as a median and interquartile range (IQR). Categorical variables were presented as percentages. In the statistical analysis, the level of $p(\alpha)<0.05$ was considered significant. Statistica 13.1 (TIBCO Inc., Palo Alto, CA, USA) and Microsoft Excel (Microsoft, Redmond, Washington, USA) were used for statistical and graphic data processing.

\section{Results}

Over a 13-year period, 66,812 invasive cardiac procedures were recorded, of which 118 were complicated by CT $(0.176 \%$ of all procedures). Depending on the procedure, the incidence of CT ranged from $0.09 \%$ to $1.42 \%$. The highest incidence was observed in the LAAC group, followed by TAVI, TPE, AFA, CIED, PCI, and EMB groups. Among 46 patients with $\mathrm{CT}$ after $\mathrm{PCl}, 29$ were initially admitted with the acute coronary syndrome (ACS). The total number of interventions and incidence of $C T$ for each group are shown in Figure 1.

The lowest median age of 55.5 (25) and 61 (8.7) was found in EMB and AFA groups, respectively. With a median age of 83.5 (5.9), the oldest patients were reported in the TAVI group. More women than men had CT after PCI, TPE, LAAC, and TAVI procedures. The most prolonged hos- 
pital stay was found in EMB 40 (59.5), LAAC 16.5 (7.5), and TPE 16 (23) groups. The LAAC and CIED patients were the most disease burdened groups, contrary to TAVI and EMB patients. At hospital admission, mild anemia was observed in TPE, LAAC, and TAVI patients, and renal impairment was mainly found in the TPE group. Baseline characteristics and data on comorbidities and laboratory parameters are summarized in Table I.

The majority of pericardial fluid drainages were performed by pericardiocentesis (104 of 118), but in 4 patients, pericardiocentesis was not satisfactory and required transport to the operation room. Overall, 16 patients had pericardiotomy, which was most often performed in the TPE group (Table II). The highest total volume of pericardial fluid was drained after LAAC (median of $925(825) \mathrm{ml}$ ) and TPE procedures (median of 580 $(700) \mathrm{ml}$ ). The lowest volumes of fluid were drained after PCls (median of $270(120) \mathrm{ml}$ ).

Inotropes were often used in patients after TAVI, while no one required inotropes in the EMB group. Blood transfusion was performed in 25-73\% (mean: 45\%) of patients. The median volume of red blood cell concentrate (RBC) was $560-8275 \mathrm{ml}$, with the most significant volumes after TAVI and EMB - 1650 and $8275 \mathrm{ml}$ of RBC, respectively (Table II).

Total in-hospital mortality was 9\%, 30-day mortality $10 \%$, and total 1 -year mortality was $14.4 \%$. The highest in-hospital mortality was observed after TAVI, EMB, and

Table I. Baseline characteristics and laboratory parameters of patients with CT complicating selected invasive cardiac procedures, from January 2006 to December 2018

\begin{tabular}{|c|c|c|c|c|c|c|c|}
\hline Parameter & $\begin{array}{c}\text { AFA } \\
(n=25)\end{array}$ & $\begin{array}{c}\mathrm{PCl} \\
(n=46)\end{array}$ & $\begin{array}{c}\text { CIED } \\
(n=25)\end{array}$ & $\begin{array}{c}\text { TPE } \\
(n=11) \\
\end{array}$ & $\begin{array}{l}\text { LAAC } \\
(n=4)\end{array}$ & $\begin{array}{c}\text { TAVI } \\
(n=3)\end{array}$ & $\begin{array}{c}\text { EMB } \\
(n=4)\end{array}$ \\
\hline Age [years] & $61.3(8.7)$ & 69 (10.6) & $73(15)$ & $76(16)$ & $74(1.8)$ & $83.5(5.9)$ & $55.5(25)$ \\
\hline Hospitalization [days] & $10(7)$ & $11(10)$ & $14(15)$ & $16(23)$ & $16.5(7.5)$ & $10(30)$ & $40(59.5)$ \\
\hline No. of women (\%) & $11(44)$ & $25(54)$ & $8(32)$ & $8(73)$ & $3(75)$ & $3(100)$ & $0(0)$ \\
\hline \multicolumn{8}{|l|}{ (\%) Disease burden: } \\
\hline Prior CAD & 24 & 49 & 44 & 36 & 50 & 33 & 0 \\
\hline Prior AF & 100 & 13 & 32 & 45 & 100 & 0 & 33 \\
\hline $\mathrm{CHF}$ & 12 & 11 & 44 & 18 & 50 & 0 & 66 \\
\hline Prior Ml & 8 & 35 & 28 & 18 & 50 & 33 & 0 \\
\hline Prior CS & 8 & 2 & 0 & 0 & 0 & 0 & 0 \\
\hline Prior PAD & 8 & 16 & 12 & 0 & 25 & 0 & 0 \\
\hline Prior CKD & 4 & 6 & 28 & 9 & 25 & 0 & 0 \\
\hline Hypertension & 48 & 64 & 48 & 27 & 50 & 0 & 0 \\
\hline Diabetes & 16 & 20 & 24 & 18 & 0 & 0 & 0 \\
\hline Hypercholesterolemia & 16 & 31 & 12 & 18 & 50 & 0 & 0 \\
\hline Obesity & 8 & 9 & 0 & 9 & 0 & 0 & 0 \\
\hline Tobacco use & 8 & 9 & 8 & 9 & 0 & 0 & 33 \\
\hline $\begin{array}{l}\text { Mean } \% \text { of all } \\
\text { comorbidities }\end{array}$ & 23 & 23 & 25 & 18 & 36 & 6 & 12 \\
\hline \multicolumn{8}{|l|}{ Laboratory parameters: } \\
\hline $\begin{array}{l}\text { Patients' data available } \\
(\%)\end{array}$ & $84 \%$ & $93-97 \%$ & $\begin{array}{c}100 \% \\
(80 \% \text { for GFR) }\end{array}$ & $\begin{array}{c}100 \% \\
\text { (81\% for GFR) }\end{array}$ & $100 \%$ & $100 \%$ & $100 \%$ \\
\hline APTT & $34.90(5.5)$ & $38.50(80.2)$ & $34.80(10.6)$ & $40.50(29.3)$ & $31.9(2.42)$ & $142.9(194)$ & $38.10(12.5)$ \\
\hline INR & $1.02(0.2)$ & $1.03(0.14)$ & $1.15(0.48)$ & $1.15(0.16)$ & $0.95(0.11)$ & $1.0(0.26)$ & $1.0(0.11)$ \\
\hline PT & $13.4(2.2)$ & $13.00(1.39)$ & $14.00(5.4)$ & $14.50(2.0)$ & $12.5(1.84)$ & $12.8(3.57)$ & $13.6(1.2)$ \\
\hline GFR & $60.00(24.9)$ & $64.87(33.95)$ & $59.50(42.69)$ & 35.00 (16.18) & $63.50(30.1)$ & $62.00(87.6)$ & 89.18 (18.6) \\
\hline $\mathrm{HCT}$ & $0.41(0.03)$ & $0.40(0.07)$ & $0.37(0.06)$ & $0.36(0.11)$ & $0.37(0.09)$ & $0.35(0.06)$ & $0.45(0.03)$ \\
\hline $\mathrm{HGB}[\mathrm{mmol} / \mathrm{l}]$ & $8.6(0.8)$ & $8.60(1.3)$ & $7.90(1.4)$ & $7.40(2.3)$ & $7.45(1.45)$ & $7.10(1.3)$ & $9.7(1.3)$ \\
\hline KREAT [mmol/l] & $86.9(37.8)$ & $81.0(32.8)$ & $98.0(46)$ & $118.0(67)$ & $69.0(60.86)$ & $82.0(14.87)$ & $88.74(27.9)$ \\
\hline $\mathrm{PLT}\left[\times 10^{9} / \mathrm{I}\right]$ & $202(67)$ & $207.5(67.5)$ & $179.0(82)$ & $186.5(47)$ & $252.0(70)$ & $190(26)$ & $250.0(81)$ \\
\hline $\mathrm{RBC}\left[\times 10^{12} / \mathrm{I}\right]$ & $4.57(0.25)$ & $4.56(0.68)$ & $4.03(0.74)$ & $396(1.37)$ & $4.24(1.45)$ & $3.92(0.7)$ & $47(0.46)$ \\
\hline $\mathrm{WBC}\left[\times 10^{9} / 1\right]$ & $6.54(2.93)$ & $8.57(3.56)$ & $7.60(2.45)$ & $9.45(7.38)$ & $5.915(0.74)$ & $5.62(6.04)$ & $9.51(6)$ \\
\hline
\end{tabular}

AF - atrial fibrillation, AFA - atrial fibrillation ablation, APTT - activated partial thromboplastin time, CAD - coronary artery disease, CHF - chronic heart failure, CIED - cardiovascular implantable electronic device, CKD - chronic kidney disease, CS - cardiac surgery, EMB - endomyocardial biopsy, GFR - glomerular filtration rate, HCT - hematocrit, HGB - hemoglobin, INR - international normalized ratio, KREAT - creatinine, LAAC - left atrial appendage closure, MI - myocardial infarction, $P A D$ - peripheral artery disease, $P C I$ - percutaneous coronary intervention, PLT - platelets, $P T$ - prothrombin time, RBC - red blood cells, TAVI - transcatheter aortic valve implantation, TPE - temporary pacing electrode, WBC-white blood cells. 
Table II. In-hospital management of patients with CT complicating invasive cardiac procedures, from January 2006 to December 2018

\begin{tabular}{|c|c|c|c|c|c|c|c|}
\hline Parameter & $\begin{array}{c}\text { AFA } \\
(n=25)\end{array}$ & $\begin{array}{c}\mathrm{PCl} \\
(n=46)\end{array}$ & $\begin{array}{c}\text { CIED } \\
(n=25)\end{array}$ & $\begin{array}{c}\text { TPE } \\
(n=11)\end{array}$ & $\begin{array}{l}\text { LAAC } \\
(n=4)\end{array}$ & $\begin{array}{c}\text { TAVI } \\
(n=3)\end{array}$ & $\begin{array}{c}\text { EMB } \\
(n=4)\end{array}$ \\
\hline No. of PCC (\%) & $18(72)$ & $39(85)$ & $22(88)$ & $8(73)$ & $3(100)$ & $4(100)$ & $4(100)$ \\
\hline $\begin{array}{l}\text { No. of surgical treat- } \\
\text { ments (\%) }\end{array}$ & $4(16)$ & $7(15)$ & $2(8)$ & $3(27)$ & 0 & 0 & 0 \\
\hline No. of PCC + surgery & $3(12)$ & 0 & $1(4)$ & 0 & 0 & 0 & 0 \\
\hline $\begin{array}{l}\text { Data available in \% } \\
\text { of patients }\end{array}$ & 72 & 74 & 76 & 45 & 100 & 33 & 50 \\
\hline $\begin{array}{l}\text { Drained pericardial } \\
\text { fluid first access [ml] }\end{array}$ & $430(400)$ & 275 (120) & $525(300)$ & $500(820)$ & $490(200)$ & $400(0)$ & $325(150)$ \\
\hline $\begin{array}{l}\text { Drained pericardial } \\
\text { fluid total }[\mathrm{ml}]\end{array}$ & $432(440)$ & $282(270)$ & $500(430)$ & $580(700)$ & 925 (825) & $400(0)$ & $325(150)$ \\
\hline Inotropes (\%) & 32 & 26 & 20 & 45 & 25 & 66 & 0 \\
\hline MVS (\%) & 4 & 19 & 4 & 45 & 25 & 33 & 0 \\
\hline $\begin{array}{l}\text { No. of } \mathrm{RBC} \text { recipients } \\
(\%)\end{array}$ & $12(48)$ & $24(52)$ & $12(48)$ & $8(73)$ & 0 & $2(66)$ & $1(25)$ \\
\hline $\begin{array}{l}\text { RBC concentrate } \\
\text { transfused [ml] }\end{array}$ & $1120(550)$ & $675(700)$ & $560(0)$ & $560(840)$ & 0 & $1650(1120)$ & $8275(0)$ \\
\hline
\end{tabular}

AFA - atrial fibrillation ablation, CIED - cardiovascular implantable electronic device, EMB - endomyocardial biopsy, Inotropes - sympathomimetic amine therapy, $\angle A A C$ - left atrial appendage closure, MVS - mechanical ventilatory support, PCC - pericardiocentesis, $P C I$ - percutaneous coronary intervention, RBC - red blood cell concentrate, TAVI - transcatheter aortic valve implantation, TPE - temporary pacing electrode.

TPE procedures. None of the AFA patients or LAAC patients died during hospitalization and up to 1 year after hospital discharge. All five in-hospital deaths in the PCI group occurred in patients with ACS. A rise in deaths up to 30 days was noted only in the TPE group. The highest 1-year mortality was found in CTs after TPE, PCI, and CIED procedures. The detailed data on in-hospital, 30-day, and 1-year mortality in patients after invasive cardiac procedures complicated with $\mathrm{CT}$ are presented in Figure 2.

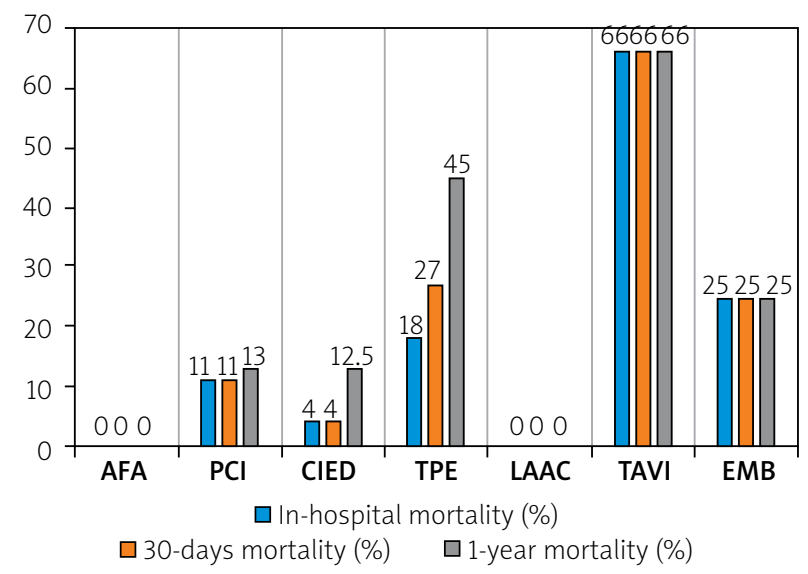

Figure 2. In-hospital, 30-day and 1-year mortality in patients after invasive cardiac procedures complicated with pericardial tamponade, from January 2006 to December 2018

AFA - atrial fibrillation ablation, CIED - cardiovascular implantable electronic device, EMB - endomyocardial biopsy, LAAC - left atrial appendage closure, $\mathrm{PCI}$ - percutaneous coronary intervention, TAVI - transcatheter aortic valve implantation, TPE - temporary pacing electrode.

\section{Discussion}

The overall rate of iatrogenic CT in published studies varies between 0.089 and $4.3 \%$, with the highest rates after TAVI, LAAC, and AFA procedures [9]. According to our analysis, the rate of CT complicating all 66,812 invasive cardiac procedures during the 13 years was $0.176 \%$. The highest CT incidence was observed in the LAAC group (1.42\%), followed by TAVI $(0.79 \%)$, TPE (0.67\%), AFA (0.45\%), CIED $(0.20 \%), \mathrm{PCl}(0.11 \%)$ and EMB $(0.09 \%)$ groups. The procedures were performed in highly experienced clinical departments of cardiology of SCCS, and hence the study results may show a relatively low risk of CT.

Many studies concerning invasive cardiac procedures have proven a correlation between female sex and the tendency to complications, including CT [10-12]. In our study, CT was more frequent among women in $\mathrm{PCI}$, TPE, LAAC, and TAVI groups. Contrary to the studies mentioned above, CT after AFA developed most often in men (56\%).

For patients at high risk of bleeding or contraindication for anticoagulation, LAAC is an alternative strategy for the mechanical prevention of thromboembolic events. Data on periprocedural major complications and long-term follow-up from large cohorts are limited [13]. In published studies where about 180 LAACs were performed, CT was seen in 1.1-2.7\% [13, 14]. A similarly high number (281) of performed LAAC procedures was recorded with the $\mathrm{CT}$ incidence of $1.42 \%$ in our cohort.

Catheter-based valve implantation has emerged as an effective treatment approach for these patients, either at very high risk for conventional surgery or even deemed inoperable [15]. In a multicenter study by Hamm et al. [15], observing prospectively 3,876 TAVI procedures, 
$1.4 \%$ of CTs were found as a post-procedural complication. Our study included 380 TAVI procedures with an incidence of $\mathrm{CT}$ of $0.79 \%$.

The evolution of catheter-based AF-ablation techniques was observed, which was considered a cornerstone of atrial fibrillation treatment [16]. Cardiac tamponade is considered the most frequent procedure-related complication, reported to occur at a rate of $0.18-2.8 \%[9,16]$. It is also the leading cause of procedure-related mortality, responsible for $25 \%$ of fatal cases [ 7,17$]$. In our study, there was a low incidence of CTs concerning electrophysiological procedures, even though we did not use periprocedural intracardiac echocardiography during the analyzed period: AFA (0.45\%) and CIED (0.2\%), which was similar to a multicenter retrospective study including 922,549 patients published by Moazzami et al. (0.28 \%) [18].

Temporary pacing electrode implantation is a lifesaving intervention for patients with hemodynamically unstable, life-threatening bradycardia or conduction system impairment. The most dangerous complications of TPE include heart perforation and CT [19]. In analyzing complications and outcomes after 360,000 TPEs, Metkus et al. defined the CT rate as $0.6 \%$ [20]. In our study, the CT incidence in the TPE group was $0.67 \%$, while the 30 -day and 1 -year mortality rates were $27 \%$ and $45 \%$, respectively.

Cardiac tamponade after $\mathrm{PCl}$ procedures is mostly caused by coronary artery perforation (CAP) and is frequently associated with poor outcomes, with a 3-fold increase in the death risk [21]. Calcifications of the coronary artery lesion and myocardial infarction are essential variables predicting CAP [22]. The $\mathrm{PCl}$-related $\mathrm{CT}$ rate in our analysis $(0.11 \%)$ was comparable to other studies (0.089-0.2\%) [9]. In our study, $63 \%$ of patients with CT after $\mathrm{PCl}$ were admitted due to ACS. Moreover, all in-hospital and 12-month deaths in the $\mathrm{PCl}$ group occurred in patients with ACS. It is also important to note that the periprocedural antiplatelet and anticoagulation drug strategies were changed during the study period as new drugs and guidelines were introduced. Unfortunately, detailed data on the antiplatelet or anticoagulant treatment directly before CT were not available in all patients, as some patients received drugs in the ambulance or the referring hospital.

The main indication for EMB is to diagnose myocarditis, allograft rejection, or secondary cardiomyopathies [23]. In the reports of 4,221 EMB procedures by Chimenti (2013) et al. and 3,048 EMBs described by Holzmann et al., the occurrence of CT was $0.08-0.29 \%[24,25]$. In our cohort of $4,257 \mathrm{EMBs}$, the incidence of CT was $0.09 \%$.

Although the highest volume of fluid drained during pericardiotomy was in the LAAC group (median of 925 (825) $\mathrm{ml}$ ), none of these patients required blood transfusion (BT). In contrast, in the $\mathrm{PCl}$ group with the lowest volume of drained fluid (270 (230) $\mathrm{ml}$ ), BT was indicated in $52 \%$ of patients.
Blood transfusion was required in $73 \%$ of patients after TPE. The frequent BT in TAVI patients (66\%) may be explained by the transapical approach compared to transvascular access ( $25.4 \%$ vs. $11.5 \%$ patients requiring $>2$ units of RBC), as reported in the GARY registry [15]. However, $\mathrm{CT}$ was more frequent in patients treated with the retrograde transvascular techniques than in those treated with transapical access [15, 26].

In the available studies, the prevalence rate of CT-related mortality ranged from $0 \%$ to $50 \%$ (mean: $7.3 \%$ ), with the highest rates observed after LAAC, TAVI, PCI, CIED, and the AFA procedures [9]. Moreover, CT after TPE implantations was associated with a 5 -fold increase in the risk for in-hospital death $(\mathrm{OR}=5.00,95 \% \mathrm{Cl}: 2.51-$ 9.96, $p<0.001$ ) [20]. None of the patients with CT died in the LAAC and AFA groups during hospitalization and up to 1 year after hospital discharge in our study. Significant in-hospital mortality was found in TAVI, EMB, and TPE groups. The highest 30-day and 1-year mortality rates were observed in the TPE group. It could be explained by the clinical status of patients referred for TPE, who are often in life-threatening situations, including cardiac arrest [27]. The group of CT after TAVI consisted of 3 patients, of whom 2 died during hospitalization. The LAAC group was also small, numbering 4 patients. These deaths were also included in the 30-day and 1-year mortality. For that reason, 1-year mortality was high.

In our study, the main focus was on cardiovascular comorbidities. Diseases such as hypothyroidism, cerebrovascular disease, chronic obstructive pulmonary disease, cancer, etc., were not included. However, based on our results, cardiovascular comorbidities were not associated with total mortality in iatrogenic CT patients.

During 1-year follow-up after hospital admission, all of the six deaths were recorded in patients with ACS treated with $\mathrm{PCl}$. In-hospital mortality after $\mathrm{CT}$ in the $\mathrm{PCl}$ group was $11 \%$ and may also be a consequence of ACS. It may be confirmed by the analysis of Gasior et al. [28], which was performed in the population of 4.6 million inhabitants recorded in the Silesian Cardiovascular Database (SILCARD). The study included data on myocardial infarctions reported between 2006 and 2014, with the annual number of hospital admissions varying between 9,619 and 11,045 . The standardized in-hospital mortality was $9.3-11.8 \%$, and 1-year mortality ranged between 17.8 and $21.5 \%$, which was higher than in our study (13\%). During the evaluated period in our hospital, automatic chest compression devices (such as the Lukas system), which may lead to increased CT risk in TPE or $\mathrm{PCl}$, were not used.

Our research was a retrospective analysis of medical histories starting in 2006. The period before 2013 was characterized by lacking full computerization of medical data. Some of the patients were admitted to the hospital in a critical condition (e.g., myocardial infarction), and not all medical parameters were measured or recorded. 
Moreover, some of them were self-reported by patients and were not consistent during the hospitalization (e.g., body mass or BMI). For that reason, some data were limited or unavailable, such as the precise depiction of taken pre- or periprocedural antiplatelet or anticoagulation therapy, as well as disease burden history. However, at this moment, we are completing updating of our database, which may bring more precise results in the future. The drained pericardial fluid volume is expected to be the most lacking one, which may be explained by emergency lifesaving settings of pericardiotomy. The second issue regarding the data gathering period (2006-2018) might be that ACS treatment recommendations were changed every few years, influencing the outcomes over the years. As some procedures were introduced or popularized later than in 2006, operators' learning curves may also influence the outcomes. Moreover, the sample sizes in the TAVI, LAAC, and EMB groups were small, which may have led to invalid interpretation of these groups' results. The data were gathered in a tertiary hospital, which should be taken into account when interpreting the results, as patients referred to our center are expected to be more challenging to treat. On the other hand, operators' experience and the number of procedures performed by operators are higher than in regional hospitals. The results on the length of hospital stay in the EMB group should be interpreted with caution, as the group contained patients with acute indications for EMB (such as myocarditis) and scheduled EMB after heart transplantation. Thus, length of stay might be determined by the underlying disease rather than PT itself.

\section{Conclusions}

The cardiac tamponade rate as a complication of invasive cardiac procedures in our hospital during the 13 years was $0.176 \%$. Nevertheless, many of the patients required a blood transfusion, inotropes as well as mechanical ventilatory support. The in-hospital mortality was highest in TAVI and EMB groups. Despite the low incidence and advanced treatment of iatrogenic CT, high in-hospital mortality rates are expected. As the sample size in some groups was relatively small, the conclusions regarding mortality should be interpreted with caution.

\section{Conflict of interest}

The authors declare no conflict of interest.

\section{References}

1. Orbach A, Schliamser JE, Flugelman MY, et al. Contemporary evaluation of the causes of cardiac tamponade: acute and longterm outcomes. Cardiol J 2016; 23: 57-63.

2. De Ponti R, Cappato R, Curnis A, et al. Trans-septal catheterization in the electrophysiology laboratory: data from a multicenter survey spanning 12 years. J Am Coll Cardiol 2006; 47: 1037-42.
3. Spodick DH. Acute cardiac tamponade. N Engl J Med 2003; 349: 684-90.

4. Holmes DR, Nishimura R, Fountain R, et al. latrogenic pericardial effusion and tamponade in the percutaneous intracardiac intervention era. JACC Cardiovasc Interv 2009; 2: 705-17.

5. Fejka M, Dixon SR, Safian RD, et al. diagnosis, management, and clinical outcome of cardiac tamponade complicating percutaneous coronary intervention. Am J Cardiol 2002; 90: 1183-6.

6. Cappato R, Calkins H, Chen S, et al. Delayed cardiac tamponade after radiofrequency catheter ablation of atrial fibrillation a worldwide report. JAC 2011; 58: 2696-7.

7. Cappato R, Calkins H, Chen SA, et al. Prevalence and causes of fatal outcome in catheter ablation of atrial fibrillation. J Am Coll Cardiol 2009; 53: 1798-803.

8. Gąsior M, Desperak P, Cieśla D, et al. How to effectively analyze scientific evidence in clinical practice? Rationale behind and design of an observational analytical model. Kardiol Pol 2020; 78: 577-80.

9. Adamczyk M, Wasilewski J, Niedziela J, et al. Pericardial tamponade as a complication of invasive cardiac procedures: a review of the literature. Adv Interv Cardiol 2019; 15: 394-403.

10. Fasseas P, Orford JL, Panetta CJ, et al. Incidence, correlates, management, and clinical outcome of coronary perforation: analysis of 16,298 procedures. Am Heart J 2004; 147: 140-5.

11. Ohlow MA, Lauer B, Brunelli $M$, et al. Incidence and predictors of pericardial effusion after permanent heart rhythm device implantation. Circ J 2013; 77: 975-81.

12. Michowitz $\mathrm{Y}$, Rahkovich $\mathrm{M}$, Oral $\mathrm{H}$, et al. Effects of sex on the incidence of cardiac tamponade after catheter ablation of atrial fibrillation: results from a worldwide survey in 34943 atrial fibrillation ablation procedures. Circ Arrhythm Electrophysiol 2014; 7: 274-80.

13. Berti S, Pastormerlo LE, Rezzaghi M, et al. Left atrial appendage occlusion in high-risk patients with non-valvular atrial fibrillation. Heart 2016; 102: 1969-73.

14. Matsuo $\mathrm{Y}$, Sandri M, Mangner N, et al. Interventional closure of the left atrial appendage for stroke prevention. Circ J 2014; 78 : 619-24.

15. Hamm CW, Mollmann H, Holzhey D, et al. The German Aortic Valve Registry (GARY): in-hospital outcome. Eur Heart J 2014; 35: 1588-98.

16. Calkins H, Hindricks G, Cappato R, et al. 2017 HRS/EHRA/ECAS/ APHRS/SOLAECE expert consensus statement on catheter and surgical ablation of atrial fibrillation: executive summary. Hear Rhythm 2017; 14: e445-94.

17. Belhassen B. A 1 per 1,000 mortality rate after catheter ablation of atrial fibrillation an acceptable risk? J Am Coll Cardiol. 2009; 12: 1804-6.

18. Moazzami K, Dolmatova E, Kothari N, et al. Trends in cardiac tamponade among recipients of permanent pacemakers in the United States: From 2008 to 2012. JACC Clin Electrophysiol 2017; 3: 41-6.

19. Erol MK, Sevimli S, Ates A. Images in cardiology: pericardial tamponade caused by transvenous temporary endocardial pacing. Heart 2005; 91: 459.

20. Metkus TS, Schulman SP, Marine JE, et al. Complications and outcomes of temporary transvenous pacing. Chest 2019; 1555 : 749-57.

21. Stathopoulos I, Kossidas K, Panagopoulos G, et al. Cardiac tamponade complicating coronary perforation during angioplasty: 
short-term outcomes and long-term survival. I Invasive Cardiol 2013; 25: 486-91.

22. Shimony A, Zahger D, Van Straten M, et al. Incidence, risk factors, management and outcomes of coronary artery perforation during percutaneous coronary intervention. Am J Cardiol 2009; 104: 1674-7.

23. Veinot JP. Diagnostic endomyocardial biopsy pathology: secondary myocardial diseases and other clinical indications - a review. Canad J Cardiol 2002; 18: 287-96.

24. Chimenti C, Frustaci A. Contribution and risks of left ventricular endomyocardial biopsy in patients with cardiomyopathies: a retrospective study over a 28-year period. Circulation 2013; 128: 1531-41.

25. Holzmann M, Nicko A, Kühl U, et al. Complication rate of right ventricular endomyocardial biopsy via the femoral approach: a retrospective and prospective study analyzing 3048 diagnostic procedures over an 11-year period. Circulation 2008; 118: 1722-8.

26. Rezq A, Basavarajaiah S, Latib A, et al. Incidence, management, and outcomes of cardiac tamponade during transcatheter aortic valve implantation. JACC Cardiovasc Interv 2012; 5: 1264-72.

27. Tjong FVY, de Ruijter UW, Beurskens NEG, et al. A comprehensive scoping review on transvenous temporary pacing therapy. Netherlands Heart J 2019; 27: 462-73.

28. Gasior M, Pres D, Wojakowski W, et al. Causes of hospitalization and prognosis in patients with cardiovascular diseases Secular trends in the years 2006-2014 according to the SILesian CARDiovascular (SILCARD) database. Pol Arch Med Wewn 2016; 126: 754-62. 